\title{
Sodium Bicarbonate Containing Mouthwash for Preventing Radiotherapy-Induced Oral Mucositis in Patients With Locally Advanced Head and Neck Cancer
}

\author{
Mehrsa Majdaeen, ${ }^{1}$ Mohammad Babaei, ${ }^{2, *}$ and Abbas Rahimi ${ }^{1}$

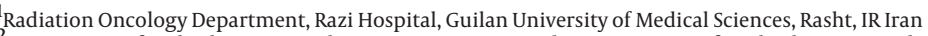 \\ ${ }^{2}$ Department of Radiotherapy Oncology, Cancer Institute, Tehran University of Medical Sciences, Tehran, IR Iran \\ *Corresponding author: Mohammad Babaei, Department of Radiotherapy Oncology, Cancer Institute, Tehran University of Medical Sciences, Tehran, IR Iran. \\ E-mail: m-babaei@sina.tums.ac.ir \\ Received: April 12, 2015; Revised: April 29, 2015; Accepted: May 28, 2015
}

\begin{abstract}
Background: In spite of all complications and disabilities due to advanced head and neck malignancies, treatment of oral mucositis following radiotherapy is mainly supportive and oral hygiene is the mainstay of treatment. In this context, mouthwash containing sodium bicarbonate can have a major role in the treatment of this complication.

Objectives: The present trial assessed the value of this type of mouthwash for preventing oral mucositis following radiotherapy in patients suffered locally advanced head and neck squamous cell carcinoma.

Patients and Methods: Twenty nine patients suffered locally advanced squamous cell carcinoma were consecutively entered into the study. All patients had stage III to IV head and neck malignancies. The patients were randomly assigned to receiving bicarbonate mouthwash $(20 \mathrm{ml}$ every 6 hours per day from the beginning to the end of the treatment $)(n=18)$ or placebo $(n=11)$. The mouthwash assessed was containing 1 vial of sodium bicarbonate, $500 \mathrm{ml}$ of normal saline, and diphenhydramine $25 \mathrm{mg} / \mathrm{kg}$ ).

Results: A significant difference was revealed in the mucositis scoring between the intervention and placebo group so that mucositis grade III or more was observed in 7 patients in intervention group (38.9\%) and in 9 patients in control group (81.8\%) that the observed discrepant was significant $(p=0.024)$. The first evidences of oral mucositis in intervention group was found in forth weeks of treatment (sessions of 16 to 17 of treatment schedule), while the appearance of mucositis in placebo group was occurred in the middle of the third week (sessions of 13 to 14 of treatment).

Conclusions: Oral care by mouthwash containing sodium bicarbonate for head and neck cancer patients undergoing radiotherapy is an effective interventional option to prevent oral mucositis.
\end{abstract}

Keywords: Mouthwash; Sodium Bicarbonate; Radiotherapy; Mucositis

\section{Background}

Oral mucositis as the painful particular inflammation and ulceration of the mucous membranes is a common and debilitating complication of chemotherapy and radiotherapy treatment for various types of cancers (1). This adverse phenomenon is generally observed in up to $80 \%$ of patients with malignancies of the head and neck receiving radiotherapy that can be more appeared in those who receiving chemotherapy (2). Furthermore, radiotherapy to the head and neck is associated with grade 3 to 4 oral mucositis, often exceeding $50 \%$ of patients (3). In this regard and for preventing its-related morbidity and, alimentary tract mucositis contributes to considerably rising health care costs (4). Also, appearing this complication can adversely affect patients' quality of life because leading to pain, loss of taste perception, decreased oral function, predisposing to various viral, bacterial, and fungal infection, and even weight loss (5). In spite of all pointed complications and disabilities, treatment of this event is mainly supportive and oral hygiene is the mainstay of treatment. In this context, salty mouthwashes have a major role in the treatment (6-8). The mouthwash containing sodium bicarbonate has been recently attention because of its revealed efficacy to relieve mucositisinduced discomfort. In a study by Turhal et al. compounded mouthwash including diphenhydramine, sodium bicarbonate, and normal saline provided effective symptomatic relief in patients with chemotherapy-induced mucositis (9). In another study by Dodd et al. comparing efficacy of mouthwash containing sodium bicarbonate versus micronized sucralfate showed no significant differences in the time to heal from the radiotherapyinduced mucositis and thus the use of this mouthwash was introduced as prudent and cost-effective method for healing mucositis (10). 


\section{Objectives}

In line with the previous studies and for clearing effect of bicarbonate mouthwashes, the present trial was performed to assess the value of these mouthwashes for preventing oral mucositis following radiotherapy in patients suffered head and neck locally advanced head and neck squamous cell carcinoma.

\section{Patients and Methods}

This study was a randomized double-blinded controlled trial approved by the ethics committee at the Guilan university of medical sciences. Twenty nine patients ( 21 men and 8 women with age ranged 44 to 68 years) suffered locally advanced squamous cell carcinoma were consecutively entered into the study. All patients had stage III to IV head and neck malignancies according to TNM classification. All patients were scheduled for receiving chemoradiotherapy at cancer department of Razi hospital in Rasht city between January 2010 and May 2011. The patients with prior radiochemotherapy, those with diabetes mellitus, thyroid disorders, patients with allergy to bicarbonate, patients using any other mouthwashes, pregnant women, and those who were required to other medications including antibiotics or analgesics could not be included. The planned radiation dose was equal to 70 Gy delivered in 35 fractions given 5 days a week concomitantly with weekly chemotherapy using cisplatin. The patients were randomly assigned to receiving bicarbonate mouthwash $(20 \mathrm{ml}$ every 6 hours per day from the beginning to the end of the treatment) $(n=18)$ or placebo $(n=11)$. The mouthwash assessed was containing 1 vial of sodium bicarbonate, $500 \mathrm{ml}$ of normal saline, and diphenhydramine $25 \mathrm{mg} / \mathrm{kg}$ ). Patients were weekly examined and blood tests were performed. The study endpoint was comparing the incidence of Mucositis grade III or higher between the interventional and placebo group according to the World Health Organization criteria for grading of oral Mucositis. Onset of Mucositis was defined as the time when the patient showed first evidence of Mucositis. Results were presented as absolute frequencies and percentages for categorical variables. Categorical variables were compared using chi-square test or Fisher's exact test when more than $20 \%$ of cells with expected count of less than 5 were observed. Statistical significance was determined as a p value of $\leq 0.05$. All statistical analysis was performed using SPSS software (version 16.0, SPSS Inc., Chicago, Illinois).

\section{Results}

A significant difference was revealed in the mucositis scoring between the intervention and placebo group so that mucositis grade III or more was observed in 7 patients in intervention group (38.9\%) and in 9 patients in control group (81.8\%) that the observed discrepant was significant ( $\mathrm{P}=$ $0.024)$. The first evidences of oral mucositis in intervention group was found in forth weeks of treatment (sessions of 16 to 17 of treatment schedule), while the appearance of mucositis in placebo group was occurred in the middle of the third week (sessions of 13 to 14 of treatment). Thus, intervention group had mucositis onset later than placebo group.

\section{Discussion}

It is a general agreement that palliative management is the optimal approach for improving radiotherapy-related oral mucositis and its complications (3). This management should be primarily begun with oral hygiene, adequate hydration for preventing dehydration, and controlling pain (11). The first step for this aim is oral hygiene that can be effectively prevent onset of mucositis that can be achieved using proper mouthwashes and adherence to soft diets (12). In this regard, mouth cleaning every four hours and at bedtime is strongly recommended. In addition, patients should check their mouth and teeth regularly to assess efficacy of the used mouthwashes. Nowadays, pharmacists are tended to modify the compounded preparations to help the patient be more compliant (13). Therefore, recent mouthwashes have been contained with some commercial drug products or active pharmaceutical ingredients such as diphenhydramine compound. Thus, we performed the present study to evaluate efficacy of one of these mouthwashes containing sodium bicarbonate in normal saline in combination with diphenhydramine. Our study showed higher preventive role of this mouthwash and its more effectiveness on preventing onset of mucositis compared with placebo group. In this compound, sodium bicarbonate provides deodorizing and buffering activities and contributes a clean mouth feel and refreshing after taste in oral cavity. In fact, sodium bicarbonate can neutralize the production of acid in the mouth and also as an antiseptic to help prevent infections occurring (14). It seems that through preventing progression of infection or inhibiting some inflammatory triggers in oral cavity, sodium bicarbonate can effectively act as a main material for preventing mucositis or can lead to delay appearance of mucositis in head and neck malignancies under radiotherapy.

One of the main advantages of this mouthwash is that can be prepared at home by own patients and therefore it is very cost-effective. According to some recommendations in guidelines, by dissolving $\frac{1}{2}$ teaspoon of sodium bicarbonate or 'baking soda' in $250 \mathrm{ml}$ of water, an appropriate mouthwash including sodium bicarbonate can be prepared (14). Beside of antiseptic effects of sodium bicarbonate, the beneficial effects of our studied mouthwash can be explained by anti-inflammatory effect of diphenhydramine. In spite of our findings on high effectiveness of bicarbonate mouthwash on preventing oral mucositis in head and neck malignancies, further studies with larger sample size and longterm follow-up of patients is recommended.

In conclusion, oral care by mouthwash containing sodium bicarbonate for head and neck cancer patients undergoing radiotherapy is an effective interventional option to prevent oral Mucositis. 


\section{References}

1. Gibson RJ, Keefe DM, Lalla RV, Bateman E, Blijlevens N, Fijlstra $M$, et al. Systematic review of agents for the management of gastrointestinal mucositis in cancer patients. Support Care Cancer. 2013;21(1):313-26.

2. Zur E. Oral mucositis: etiology, and clinical and pharmaceutical management. Int J Pharm Compd. 2012;16(1):22-33.

3. Caillot E, Denis F. [Radio-induced oral and pharyngeal mucositis: management updates]. Cancer Radiother. 2012;16(56):358-63.

4. Sonis ST. Oral mucositis in cancer therapy. J Support Oncol. 2004;2(6 Suppl 3):3-8.

5. Carl W, Havens J. The cancer patient with severe mucositis. Curr Rev Pain. 2000;4(3):197-202.

6. Silverman SJ. Diagnosis and management of oral mucositis. J Support Oncol. 2007;5(2 Suppl 1):13-21.

7. Medina PJ, Fausel C. Cancer treatment and chemotherapy. In: DiPiro J. T., Talbert R. L., Yee G. C., editors. Pharmacotherapy: A Pathophysiologic Approach. New York: McGraw-Hill Medical; 2008. pp. 2115-6.

8. Cornett PA, Dea TO. Cancer. In: McPhee SJ, Papadakis MA, Rabow MW, editors. Current Medical Diagnosis and Treatment 2011. New
York: McGraw-Hill Medical; 2011.

9. Turhal NS, Erdal S, Karacay S. Efficacy of treatment to relieve mucositis-induced discomfort. Support Care Cancer. 2000;8(1):55-8.

10. Dodd MJ, Miaskowski C, Greenspan D, MacPhail L, Shih AS, Shiba $\mathrm{G}$, et al. Radiation-induced mucositis: a randomized clinical trial of micronized sucralfate versus salt \& soda mouthwashes. Cancer Invest. 2003;21(1):21-33.

11. Li E, Trovato JA. New developments in management of oral mucositis in patients with head and neck cancer or receiving targeted anticancer therapies. Am J Health Sys Pharm. 2012;69(12):1031-7.

12. Rodriguez-Caballero A, Torres-Lagares D, Robles-Garcia M, Pachon-Ibanez J, Gonzalez-Padilla D, Gutierrez-Perez JL. Cancer treatment-induced oral mucositis: a critical review. Int J Oral Maxillofac Surg. 2012;41(2):225-38.

13. Shih A, Miaskowski C, Dodd MJ, Stotts NA, MacPhail L. A research review of the current treatments for radiation-induced oral mucositis in patients with head and neck cancer. Oncol Nurs Forum. 2002;29(7):1063-80.

14. Farah CS, McIntosh L, McCollough MJ. Mouthwashes. Aus Prescrib. 2009;32 (6):162-4. 
